\title{
POTENCIAL DE HIBRIDACION NATURAL EN EL MEZQUITE (PROSOPIS LAEVIGATA Y P. GLANDULOSA VAR. TORREYANA, LEGUMINOSAE) DE LA ALTIPLANICIE DE SAN LUIS POTOSI'
}

\author{
Sergio Galindo Almanza \\ Instituto Tecnológico de Los Mochis, SEP, \\ Apartado postal 766 \\ 81200 Los Mochis, Sin. México \\ Edmundo Garcia Moya, Thomas L. Wendt \\ y Felix V. Gonzalez Cossio \\ Colegio de Postgraduados, \\ Centro de Estadística y Cálculo \\ 56230 Chapingo, Edo. de Méx., México
}

\section{RESUMEN}

El objetivo de este estudio es demostrar que existe hibridación natural entre las especies de mezquite (Prosopis laevigata (Humb. \& Bonpl. ex Willd.) Johnst. y Prosopis glandulosa var. torreyana (L. Benson) Johnst.) que prosperan en la altiplanicie de San Luis Potosí. Se establece la ausencia de aislamiento interespecífico por los siguientes tipos de barreras: geográficas, ecológicas, fenológicas, agentes de polinización, cromosómicas y por incompatibilidad reproductiva. Mediante estudios de la morfologia de las plantas en poblaciones naturales se muestran evidencias de hibridación; experimentalmente se obtuvieron plantas híbridas.

\section{ABSTRACT}

The purpose of this study is to demonstrate that hybridization occurs between mesquite species (Prosopis laevigata (Humb. \& Bonpl. ex Willd.) Johnst. and Prosopis glandulosa var. torreyana (L. Benson) Johnst.) in the highlands of San Luis Potosi. We found no geographic, ecological, phenological, pollination, chromosomal or compatibility barriers to interspecific hybridization. Morphological evidence of natural hybridization was observed, and by artificial crosses hybrids were obtained.

\section{INTRODUCCION}

El mezquite es un recurso biótico con amplia distribución geográfica y ecológica en las zonas áridas mexicanas. Para las etnias nómadas precolombinas fue muy útil como fuente de alimento, combustible y medicina. En la actualidad se le considera con potencial

\footnotetext{
1 Este trabajo forma parte de la tesis con la cual el primer autor obtuviera el grado de Maestría en Ciencias en el Centro de Botánica del Colegio de Postgraduados, Chapingo, México.
} 
como forraje, material de construcción y combustible; sus comunidades proporcionan sitios para recreación humana, refugio de fauna silvestre, fuente de néctar para abejas y otros insectos, es importante también en la retención del suelo, ya que previene el proceso de desertificación (Galindo y Garcia, 1986a). Su cultivo y mejoramiento han sido recomendados por varios autores, toda vez que posee una amplia variabilidad genética intraespecífica (Burkart, 1976; Felker, 1979; Leakey y Last, 1980; Galindo, 1983; Galindo y Garcia, 1986a y b).

De este modo, uno de los aspectos que requiere atención es el referente a la hibridación natural, proceso que podría ser frecuente entre las especies del género Prosopis, pero cuya ocurrencia no se ha probado aún de modo saatisfactorio. En la altiplanicie de San Luis Potosí, Galindo (1983) encontro evidencias preliminares de su existencia, las cuales han llevado a plantear esta investigación, cuyo objetivo es el de definir si ocurre o no hibridación natural entre las especies de mezquite ( $P$. laevigata (Humb. \& Bonpl. ex Willd.) M.C. Johnst. y P. glandulosa var. torreyana (L. Benson) M.C. Johnst.) que prosperan en dicha región.

En el género Prosopis se presenta una amplia variabilidad intra e interespecifica en caracteres como: producción de frutos, morfología del fruto, hábito y tasa de crecimiento, adaptabilidad al frío, calor y salinidad (Felker, 1979; Galindo, 1983). Es claro entonces que la capacidad de hibridación sería sumamente útil en programas de mejoramiento genético para diferentes usos y hábitats. Este estudio ayudaría también a definir un adecuado tratamiento biosistemático en el género Prosopis, ya que la taxonomía de muchas de sus especies es confusa, posiblemente debido a hibridación. Además, este trabajo resultaría interesante en el ámbito de la evolución orgánica (Galindo, 1986).

\section{ANTECEDENTES}

Burkart (1952) informo sobre el hallazgo de individuos con características foliares intermedias entre especies de Prosopis de Argentina, a los cuales designan como de probable origen híbrido.

Graham (1960), mediante un estudio detallado de la variación morfológica en el mezquite de Tamaulipas y del este de Nuevo León, encontró individuos con características intermedias, lo cual sugiere la ocurrencia de hibridación entre Prosopis glandulosa Torr. var. glandulosa y $P$. laevigata.

Johnston (1962) detectó un fenómeno que denominó "contaminación de poblaciones" de $P$. glandulosa var. torreyana por genes de $P$. velutina Wooton en el sur y oeste de Coahuila. Indicó asimismo que, en los alrededores de Guaymas, Sonora y en La Paz, Baja California Sur, hay plantas de mezquite que exhiben una combinación de caracteres de $P$. articulata S. Wats., $P$. glandulosa var. torreyana, $P$. velutina y a veces aun de $P$. juliflora (Sw.) DC. Señalo también el haber encontrado indicios de hibridación y posterior introgresión en las siguientes situaciones: entre $P$. laevigata y $P$. glandulosa var. torreyana en Nueces County, Texas, y entre $P$. laevigata y $P$. glandulosa var. glandulosa en el suroeste de Nuevo León.

Según Isely (1972), en el sur de Nuevo México (Valle de la Mesilla) P. glandulosa var. glandulosa parece traslaparse en sus caracteres morfologicos con la var. torreyana. El mismo autor añade que ha colectado especímenes fenotipicamente intermedios en 
varios grados entre $P$. velutina y $P$. glandulosa var. torreyana, principalmente en el suroeste de Arizona.

Palacios y Bravo (1975) encontraron que varias especies de Prosopis pueden identificarse por la morfología de sus semillas. Sin embargo, apuntaron que no sucede asi en el caso de $P$. velutina y $P$. glandulosa con sus dos variedades, ya que las diferencias entre tales taxa no son tan constantes, hecho que se acentúa cuando los individuos crecen en simpatría. Esto les hace pensar en una posible hibridación y posterior introgresión.

Los anteriores informes de aparentes casos de hibridación en Prosopis motivaron a Solbrig et al. (1977) a realizar un estudio citologico sobre la mayoría de las especies del género. Como resultado del mismo establecieron el número cromosómico de 28 de las 44 especies de Prosopis; todos los taxa estudiados son diploides, con un número haploide de $14(2 n=28)$, excepto $P$. juliflora que parece tener razas diploides y tetraploides. También señalaron haber observado al menos ocho diferentes poblaciones en Sudamérica, que contienen híbridos aparentes, todos con alta fertilidad y características morfologicas intermedias. Concluyeron que, en términos de morfología y número cromosómico, parece haber pocas barreras genéticas y cromosómicas para la hibridación entre varias especies de Prosopis.

Burkart (1976) y Arroyo (1981) opinaron que el número cromosómico de $n=14$ es correcto para Prosopis y que recuentos diferentes y reconocimientos de tetraploides (como en $P$. juliflora y $P$. glandulosa) son probablemente incorrectos. El primer autor señaló también que la meiosis parece ser normal aun en supuestos individuos híbridos.

En adición a las características citológicas, la simpatrla, el traslapo parcial de los tiempos de floración y la poca discriminación entre especies por parte de los insectos polinizadores, facilita la hibridación (Solbrig et al., 1977). Estos autores encontraron también que las especies de mezquite de México y del suroeste de los Estados Unidos son químicamente afines, pues presentan flavonoides similares.

Benson y Darrow (1981), Felker (1981) y Felker et al. (1983) registraron probables casos de hibridación en mezquite.

Galindo (1983), al estudiar la variación morfológica en las especies de Prosopis de la altiplanicie de San Luis Potosí, hizo algunas colectas de individuos con características intermedias entre $P$. laevigata y $P$. glandulosa var. torreyana; la explicación más plausible es la ocurrencia de hibridación natural.

La gran variabilidad intraespecifica existente en los caracteres foliares de la mayoría de las especies de la sección Algarobia podría deberse, en algunos casos, a la existencia de hibridación introgresiva (Martínez, 1984).

Palacios y Bravo (1981), al analizar los compuestos fenólicos foliares, aportaron evidencias cromatográficas acerca de la ocurrencia de hibridación entre varias especies de Prosopis en Argentina. También en ese país, Naranjo et al. (1984) estudiaron tres híbridos naturales hipotéticos diferentes de Prosopis, analizándolos conjuntamente a través de la morfología, citogenética y cromatografía de fenoles. Asimismo Hunziker et al. (1986) utilizaron la cromatografía de compuestos fenólicos para la caracterización de 45 especies y la identificación de los híbridos naturales. 


\section{MATERIALES Y METODOS}

\section{Barreras geográficas}

Se precisó la distribución geográfica de Prosopis en la altiplanicie de San Luis Potosí y regiones adyacentes, con el propósito de ubicar las áreas de alopatría y de simpatría de $P$. laevigata y $P$. glandulosa var. torreyana.

Para el efecto, se procedio como sigue:

a) Se realizaron recorridos exploratorios siguiendo el trazo de las carreteras y terracerías principales.

b) Se identificaron, a intervalos regulares de 20 a $25 \mathrm{~km}$, los primeros 25 árboles que se encontraron, verificándose luego mediante un recorrido de reconocimiento, si la relación hallada era representativa. De este modo se registro la especie o especies que prosperan en cada punto de reconocimiento.

\section{Barreras ecológicas}

El cotejo de la presencia o ausencia de aislamiento por hábitat entre las especies consideradas, localizadas en simpatría, se efectuó ubicando y marcando con pintura de esmalte pares de árboles que crecían juntos (con una separación máxima de $5 \mathrm{~m}$ entre si), se marco de amarillo a $P$. laevigata y de rojo a $P$. glandulosa var. torreyana.

\section{Barreras fenológicas}

En el sitio de estudio de Salinas (Fig. 1), la corroboración de la ausencia de aislamiento fenológico se realizó mediante la observación continua de los pares de árboles marcados durante un periodo de floración.

4. Barreras reproductivas determinadas por agentes de polinización

Con el propósito de indagar si los polinizadores son especialistas o generalistas, se observó la forma en que los insectos recolectaron el néctar y polen; posteriormente se capturaron e identificaron los insectos visitantes de las inflorescencias de los árboles marcados en el sitio de estudio.

\section{Barreras cromosómicas}

La corroboración del número cromosómico de las dos especies de mezquite, se realizó de acuerdo con la metodología recomendada por Patiño (1981). Para ello se hicieron 10 colectas de botones florales de cada especie, conforme al procedimiento descrito por Love y Love (1975). 


\title{
6. Barreras por incompatibilidad reproductiva
}

Para constatar si existe o no incompatibilidad reproductiva se efectuaron las siguientes polinizaciones artificiales en los árboles marcados; la especie colocada en primer término funcionó como receptora del polen de la segunda:

\author{
$P$. laevigata $\times P$. laevigata (autogamia) \\ $P$. laevigata $X P$. laevigata (alogamia) \\ $P$. glandulosa var. torreyana $X P$. glandulosa var. torreyana (autogamia) \\ $P$. glandulosa var. torreyana $X P$. glandulosa var. torreyana (alogamia) \\ $P$. laevigata $X P$. glandulosa var. torreyana \\ $P$. glandulosa var. torreyana $X P$. laevigata
}

Previo a los tratamientos, se procedió al aislamiento de inflorescencias y obtención de polen. Para tal fin algunas espigas se cubrieron en estado de botón con bolsas de glassine perforadas con una aguja de disección con el objeto de evitar sobrecalentamiento en el ambiente interno. Al mismo tiempo se colectaron inflorescencias con flores en antesis, en las cuales se estimuló la dehiscencia de las anteras mediante la exposición por 48 horas a una temperatura aproximada de $25^{\circ} \mathrm{C}$ bajo una lámpara de luz incandescente. Una vez seco, el material se tamizó a diferentes tamaños de poros hasta obtener el polen, mismo que se colocó en un tubo vial que, cubierto con una torunda, se almacenó a una temperatura de aproximadamente $5^{\circ} \mathrm{C}$.

Para efectuar las cruzas artificiales se procedio a la emasculación del primer grupo de flores (un promedio de 30 ) que alcanzaban la antesis en las inflorescencias aisladas (el resto de las flores se eliminó); enseguida se realizó la polinización artificial con polen almacenado. Las inflorescencias tratadas se cubrieron nuevamente y se observaron los resultados cinco días después, cuando el pequeño fruto ya puede reconocerse como una unidad evidente de aproximadamente $1 \mathrm{~cm}$ de largo.

Como testigo, se aplicaron los siguientes tratamientos:

Tratamiento testigo A. Inflorescencias que aisladas desde el estado de botón (mediante una bolsa de glassine), se descubren al inicio de la antesis; una vez que las flores en antesis son emasculadas y que se eliminan los demás botones florales, la inflorescencia se cubre de nuevo.

Tratamiento testigo B. Inflorescencias que son aisladas desde el estado de botón, y que se descubren cuando las primeras flores alcanzan la antesis, siendo estas emasculadas; después de eliminar los restantes botones florales, las espigas son dejadas a la intemperie.

Tratamiento testigo C. Inflorescencias que permanecen aisladas mediante la bolsa de glassine, desde el estado de botón.

Del resultado de las cruzas se verificó (cuando fue posible) el estado de desarrollo de las semillas y la viabilidad de los híbridos mediante pruebas de germinación en macetas, ya que pueden ser otros los mecanismos de aislamiento que impedirian en el futuro el éxito del establecimiento de la progenie. 


\section{Evidencia morfológica de la hibridación}

Se hicieron colectas en 228 individuos de poblaciones de mezquite de los sitios previamente seleccionados (Fig. 1). De dichos individuos, 60 pertenecen a 4 poblaciones casi puras de Prosopis laevigata, 52 a una población en igual condición de $P$. glandulosa var. torreyana, 75 a poblaciones mixtas de las dos especies anteriores, y 33 a una población pura de $P$. glandulosa var. glandulosa; los restantes 8 individuos corresponden a supuestos híbridos con caracteristicas foliares intermedias.

Las colectas se realizaron por el método de los cuadrantes centrados en un punto (Mueller-Dombois y Ellenberg, 1974), considerando individuos con frutos. No obstante, en el sitio de Salinas, se seleccionaron 8 individuos, apoyándose en la metodologla propuesta por Goodman (1974).

De cada uno de los 228 individuos de mezquite se tomaron dos muestras, de las que, a partir de una hoja madura, se registraron las siguientes variables:

$\mathrm{V}_{1}=$ Número de pares de pinnas por hoja

$V_{2}=$ Número de pares de foliolos por pinna

$V_{3}=$ Longitud del foliolo $(\mathrm{mm})$

$V_{4}=$ Anchura del foliolo $(\mathrm{mm})$

$\mathrm{V}_{5}=$ Distancia interfoliolo $(\mathrm{mm})$

$\mathrm{V}_{6}=$ Longitud del peciolo $(\mathrm{cm})$

$\mathrm{V}_{7}=$ Longitud del raquis primario $(\mathrm{cm})$

$\mathrm{V}_{\mathrm{B}}=$ Longitud total de la hoja $(\mathrm{cm})$

Estos datos fueron sometidos a un análisis clasificatorio discriminante, el cual se describe a continuación.

El análisis clasificatorio discriminante es un método estadístico muy útil en taxonomía numérica que usa la "distancia cuadrada generalizada" como medida de afinidad para distinguir entre grupos, o para establecer la más probable población parental para un individuo o grupo; permite asimismo probar la integridad de los grupos definidos antes del análisis (Orloci, 1978).

Para un conjunto de observaciones que contiene una o más variables cuantitativas y una clasificación variable que define grupos de observaciones, el análisis clasificatorio discriminante desarrolla una función para clasificar cada observación dentro de uno de los grupos; se asume que los grupos tienen una distribución normal multivariada. Dicha función discriminante, que es determinada por una medida de "distancia cuadrada generalizada", se basa en las matrices de covarianza individuales dentro de cada grupo o bien en una matriz de covarianza conjunta. Una vez que se obtiene la función, cada observación se colocará en el grupo con el que tenga la distancia cuadrada generalizada más pequeña, ya que ésta es una estimación de semejanza que refleja la distancia taxonómica (Anónimo, 1982; Sokal y Sneath, 1963; Jardine y Sibson, 1971).

En este análisis también puede aplicarse una probabilidad umbral, la cual especifica una probabilidad mínima aceptable para clasificación, de manera que si ésta es menor que el valor umbral asignado previamente, la observación es clasificada en un grupo denominado "otros" (Anónimo, 1982). 


\section{RESULTADOS Y DISCUSION}

\section{Barreras geográficas en las especies de Prosopis}

Al definir la distribución de las especies de Prosopis en la altiplanicie potosina y áreas adyacentes (Fig. 2), se encuentra que las poblaciones puras de Prosopis laevigata prosperan en el extremo sur de este territorio, y se continúan hacia el este, extendiéndose en gran parte del estado de San Luis Potosí y sur de Nuevo León. Las poblaciones puras de $P$. glandulosa var. torreyana se localizan en el extremo norte de San Luis Potosí extendiéndose al norte de Zacatecas, sureste de Coahuila y amplias áreas adyacentes de Nuevo León. En la porción central de la altiplanicie potosina la simpatría entre las dos especies mencionadas alcanza su mejor expresión, por lo que se le considera la principal zona de hibridación potencial. En el suroeste del estado de Nuevo León se ha ubicado, asimismo, un área en la que coexisten poblaciones de $P$. glandulosa var. torreyana, $P$. laevigata y $P$. glandulosa var. glandulosa, lo que permite reafirmar el supuesto de ausencia de barreras geográficas. La existencia de las zonas de simpatría de dos o más especies de Prosopis ha sido señalada por diversos autores (Burkart, 1952; Graham, 1960; Johnston, 1962; Isely, 1972, Benson y Darrow, 1981; Palacios y Bravo, 1981) y es considerada como condición en la posibilidad de hibridación.

\section{Barreras ecológicas}

La simpatría entre $P$. laevigata y $P$. glandulosa var. torreyana, no es sólo geográfica sino también ecológica o de hábitat, pues los individuos de una especie crecen al lado de los de la otra compartiendo un mismo hábitat, lo que implica que se hallan bajo la influencia de los mismos factores bióticos y físicos, y, que por lo tanto, las especies no presentan barreras ecológicas. Similares conclusiones derivaron para otras especies de Prosopis, Burkart (1952), Graham (1960), Johnston (1962), Isely (1972), Benson y Darrow (1981) y Palacios y Bravo (1981).

\section{Barreras fenológicas}

Los períodos de floración de $P$. laevigata y de $P$. glandulosa var. torreyana coinciden completamente en la zona de simpatría, lo que favorece la posibilidad de hibridación entre los dos tipos de plantas.

Para explicar la angosta zona de contacto entre $P$. glandulosa var. glandulosa y $P$. laevigata en el norte de Tamaulipas y este de Nuevo León, Johnston (1962) señala que estudios realizados en el norte de la primera entidad indican que $P$. glandulosa var. glandulosa libera el polen en abril, y que $P$. laevigata, en el sur del mismo estado, lo hace principalmente en marzo, pero en la zona de contacto no hay diferencias en el tiempo de floración. Por su parte, Palacios y Bravo (1981) han observado que en la región chaqueña argentina las especies simpátricas se caracterizan por la floración simultánea; esto conduce a los mencionados autores a considerar la posibilidad de hibridación. 
4. Barreras reproductivas determinadas por agentes de polinización

Las observaciones hechas en la primavera de 1985 en el sitio de estudio de Salinas, señalan que los insectos consignados en el Cuadro 1 muestran una fuerte preferencia por las inflorescencias de Prosopis y que en su gran mayoría no hacen ninguna distinción entre las dos especies. Esto permite descartar la posibilidad del tipo de aislamiento aquí discutido. Al respecto, cabe referir la conclusión de Simpson et al. (1977), quienes deducen que en vista de que las especies de la sección Algarobia que crecen en forma simpátrica en Angalgalá (sitio ubicado en la zona árida argentina) florecen al mismo tiempo, tienen flores similares y son visitadas indiscriminadamente por abejas y avispas, existe una amplia oportunidad para la hibridación interespecifica.

Cuadro 1. Insectos voladores visitantes de las inflorescencias de P. laevigatay P. glandulosa var. torreyana colectados en el sitio de estudio de Salinas, S.L.P., en mayo de 1985. (La identificación fue hecha por N. Bautista M., del Centro de Entomología y Acarología del Colegio de Postgraduados, Chapingo, México).

\begin{tabular}{|clll|}
\hline ORDEN & FAMILIA & GENERO Y ESPECIE & ABUNDANCIA RELATIVA \\
\hline Hymenoptera & Megachilidae & Ashmaediella sp. & abundante \\
Hymenoptera & Megachilidae & Calicodoma sp.* & frecuente \\
Hymenoptera & Megachilidae & Calicodoma sp. & abundante \\
Hymenoptera & Megachilidae & no identificado* & frecuente \\
Hymenoptera & Megachilidae & no identificado* & frecuente \\
Hymenoptera & Megachilidae & no identificado* & frecuente \\
Hymenoptera & Colletidae & Coletes sp. & abundante \\
Hymenoptera & Colletidae & no identificado* & escasa \\
Hymenoptera & Colletidae & no identificado* & frecuente \\
Hymenoptera & Anthophoridae & no identificado* & frecuente \\
Hymenoptera & Anthophoridae & no identificado* & escasa \\
Hymenoptera & Anthophoridae & no identificado* & escasa \\
Hymenoptera & Apidae & Apis mellifera* & abundante \\
Hymenoptera & Bombidae & Bombus pennsylvanicus" & escasa \\
Hymenoptera & Vespidae & no identificado** & frecuente \\
Hymenoptera & Vespidae & no identificado* & escasa \\
Hymenoptera & Shpecidae & no identificado* & escasa \\
Diptera & Syrphidae & Copestilum mexicanum & escasa \\
Diptera & Syrphidae & no identificado* & frecuente \\
Diptera & Syrphideae & no identificado* & escasa \\
Diptera & Syrphidae & Eristalis sp.* & frecuente \\
Diptera & Sarcophagidae & Sarcophagia sp.* & escasa \\
\hline
\end{tabular}

"Especies que favorecen la polinización cruzada interespecifica por su conducta de forrajeo. 


\section{Barreras cromosómicas}

Los recuentos cromosómicos hechos en el material proveniente de cinco individuos de $P$. laevigata, revelaron 14 cromosomas en la condición haploide y, por tanto, un número diploide de 28. Esto concuerda con lo encontrado por Solbrig et al. (1977) para la misma especie. El material colectado de $P$. glandulosa var. torreyana no permitió hacer ningún recuento. No obstante, se dispone de la información consignada por Solbrig et al. (1977) que indica también un número cromosómico diploide de 28 para esta variedad.

Los resultados aquí consignados, junto con la conocida uniformidad en número y morfología cromosómica en 28 de las especies de Prosopis, y el comportamiento meiótico normal en algunos híbridos hipotéticos, nos hace coincidir con autores como Burkart (1976), Solbrig et al. (1977), Arroyo (1981), Palacios y Bravo (1981), Naranjo et al. (1984) y Hunziker et al. (1986), quienes han inferido la ausencia de barreras cromosómicas entre las especies afines del género.

\section{Barreras por incompatibilidad reproductiva}

En los cruzamientos siguientes, los valores de fecundación que se indican, son expresiones porcentuales que resultan al considerar el número de flores polinizadas por inflorescencia y el número de frutos iniciales (aquellos que se perciben cinco días después de la fecundación).

\subsection{Cruzamientos autógamos}

Los porcentajes de fecundación resultantes de autopolinizar ocho inflorescencias de 2 árboles de Prosopis laevigata variaron entre 65.6 y $85.0 \%$; y los observados en 5 inflorescencias de un individuo de $P$. glandulosa var. torreyana se ubicaron entre 26.3 y $78.9 \%$.

\subsection{Cruzamientos alógamos}

La cruza entre P. laevigata y $P$. laevigata se efectuó en 14 inflorescencias de cuatro árboles cuyas flores fueron polinizadas con polen proveniente de otro individuo. Se obtuvo entre 33.3 y $92.0 \%$ de fecundación y se cosecharon 26 frutos maduros.

El tratamiento de $P$. glandulosa var. torreyana $X$. glandulosa var. torreyana se efectuó en cinco inflorescencias de un árbol. La fecundación observada varió entre 13.3 y $80.4 \%$.

La cruza $P$. laevigata $X$. glandulosa var. torreyana se practicó en 42 inflorescencias de 10 individuos de $P$. laevigata; el polen provino de siete individuos de $P$. glandulosa var. torreyana. Hubo compatibilidad reproductiva interespecifica, con eficiencias de fecundación entre 10 y $100 \%$. Se cosecharon 91 frutos maduros.

El tratamiento $P$. glandulosa var. torreyana $X P$. laevigata se efectuó en 36 inflorescencias de 10 individuos de la primera especie, usando polen de ocho individuos de la segunda. Se presento de 30 a $100 \%$ de fecundación y se cosecharon 51 frutos maduros. 
El reducido número de frutos maduros que se cosecharon se debe a que la mayoría quedan destruídos durante su desarrollo por insectos y ganado.

La compatibilidad reproductiva observada elimina el supuesto de la existencia de este tipo de barreras. No se conocen antecedentes sobre polinizaciones artificiales interespecificas en Prosopis.

\subsection{Tratamientos testigo}

Tratamiento testigo A. Fueron sometidas a este tratamiento 20 inflorescencias ( $x$ = 30 flores, en cada una), de cinco árboles distintos de $P$. laevigata y de $P$. glandulosa var. torreyana. En ningún caso hubo fecundación, lo que indica la ausencia de cleistogamia y apomixis.

Tratamiento testigo B. Se sujetaron a estas condiciones 10 inflorescencias de $P$. laevigata y otras tantas de $P$. glandulosa var. torreyana, con un promedio de 30 flores cada una. Los porcentajes de fecundación para cada especie fueron de 3.4 y $1.8 \%$, respectivamente. Se observa que la carencia de atrayentes visuales y en parte alimenticios (polen), aportados por las anteras, hacen inconspicuas las flores a los principales polinizadores (himenópteros). Sólo pequeños hemípteros deambulaban entre ellas probablemente en busca de néctar. De esta manera los resultados ponen de manifiesto que en la polinización de Prosopis probablemente intervienen también grupos no considerados hasta ahora por autores como Simpson et al. (1977), como es el caso de hemípteros y coleópteros cuya acción provoca por lo común una polinización de tipo geitonogámico (con el polen de una flor vecina).

Tratamiento testigo C. Este tratamiento se aplicó a 37 inflorescencias de cuatro individuos de $P$. laevigata, y a 17 correspondientes a tres arbustos de $P$. glandulosa var. torreyana. En la primera especie se observaron porcentajes de autofecundación que variaron entre cero y 8.9 mientras que para la segunda, estos se ubicaron entre cero y $4.6 \%$.

En oposicion a lo registrado hasta ahora por otros autores (Signoret, 1970; Simpson et al. 1977; Arroyo, 1981; Palacios y Bravo, 1981; Karlin y Díaz, 1984; Nájera y Pimienta, 1984), en el sentido de la ampliamente extendida autoincompatibilidad en Prosopis, nosotros hemos observado que existe una capacidad de autofecundación en las especies estudiadas.

\subsection{Estado de desarrollo de las semillas}

Los frutos resultantes de los cruzamientos interespecificos tuvieron un desarrollo normal, el cual requirió de casi cuatro meses (desde la fecundación hasta su total maduración). La producción de semillas y su morfología son, asimismo, en apariencia semejantes a las generadas mediante la polinización natural.

Solbrig et al. (1977) y Palacios y Bravo (1981) observaron que los híbridos hipotéticos que identificaron presentaban un alto grado de fertilidad. Por su parte, Naranjo et al. (1984), informaron sobre tres híbridos hipotéticos de $P$. affinis $X P$. nigra que producen pocos frutos y semillas bien desarrolladas. En el caso aquí estudiado no se observó ningún indicio sobre una relación entre supuestos híbridos y la disminución de la fertilidad (reflejada en la producción de frutos). 


\subsection{Viabilidad de los híbridos}

Un total de 160 semillas, cuyos orígenes se especifican en el Cuadro 2, se pusieron en macetas con suelo húmedo proveniente de la misma área de estudio en que se obtuvieron. De las 80 semillas de origen híbrido germinaron $14(17.5 \%)$, mientras que de las 80 resultantes de polinización natural se activaron $5(6.25 \%)$. Las primeras germinaron siete dias después de su siembra. Siete meses más tarde todas las plántulas híbridas sobrevivieron y exhibieron un crecimiento y desarrollo normal (comparándolas con las que sirvieron de testigo). Hasta ese momento no hubo ninguna señal que indicara la inviabilidad de los híbridos.

Cuadro 2. Porcentajes de germinación en semillas de mezquite de origen híbrido (polinización artificial) y en semillas de las especies parentales (polinización natural), sembradas en macetas durante enero de 1986 en Salinas, S.L.P.

\begin{tabular}{|lccc|}
\hline \multicolumn{1}{|c|}{ Origen de las semillas } & $\begin{array}{l}\text { Núm. de semillas } \\
\text { sembradas que germinaron }\end{array}$ & $\begin{array}{c}\text { Porcentaje de } \\
\text { germinación }\end{array}$ \\
\hline $\begin{array}{l}\text { P. laevigata X P. glandulosa var. } \\
\text { torreyana }\end{array}$ & 40 & 9 & 22.5 \\
$\begin{array}{l}\text { P. glandulosa var. torreyana X } \\
\text { P. laevigata }\end{array}$ & 40 & 5 & 12.5 \\
$\begin{array}{l}\text { P. laevigata (poliniz. natural) } \\
\begin{array}{l}\text { P. glandulosa var. torreyana } \\
\text { (poliniz. natural) }\end{array}\end{array}$ & 40 & 5 & 12.5 \\
\hline
\end{tabular}

\section{Evidencia morfológica de la hibridación}

El propósito del análisis clasificatorio discriminativo fue discernir indirectamente sobre la ausencia de aislamiento debido a la esterilidad de híbridos F1 o F2.

En un primer análisis de la información cuantitativa referente a los caracteres foliares del total de las colectas hechas (228), se confirmaron los cinco grupos que se hablan definido previamente de acuerdo con la procedencia de las colectas. Así, el grupo 1 representa a las cuatro poblaciones casi puras de $P$. laevigata, el grupo 2 a la población pura de $P$. glandulosa var. torreyana, el grupo 3 a las poblaciones simpátricas de $P$. laevigata y $P$. glandulosa var. torreyana, el grupo 4 a la población pura de $P$. glandulosa var. glandulosa, y el grupo 5 a los ocho individuos colectados de manera selectiva debido a que mostraban de modo evidente características morfológicas intermedias entre $P$. laevigata y $P$. glandulosa var. torreyana.

Con el análisis clasificatorio discriminante cuyos resultados se presentan enseguida, se pretende conocer en el sitio de estudio de Salinas, la relación cuantitativa existente entre los individuos de las especies parentales y los posibles individuos híbridos 0 


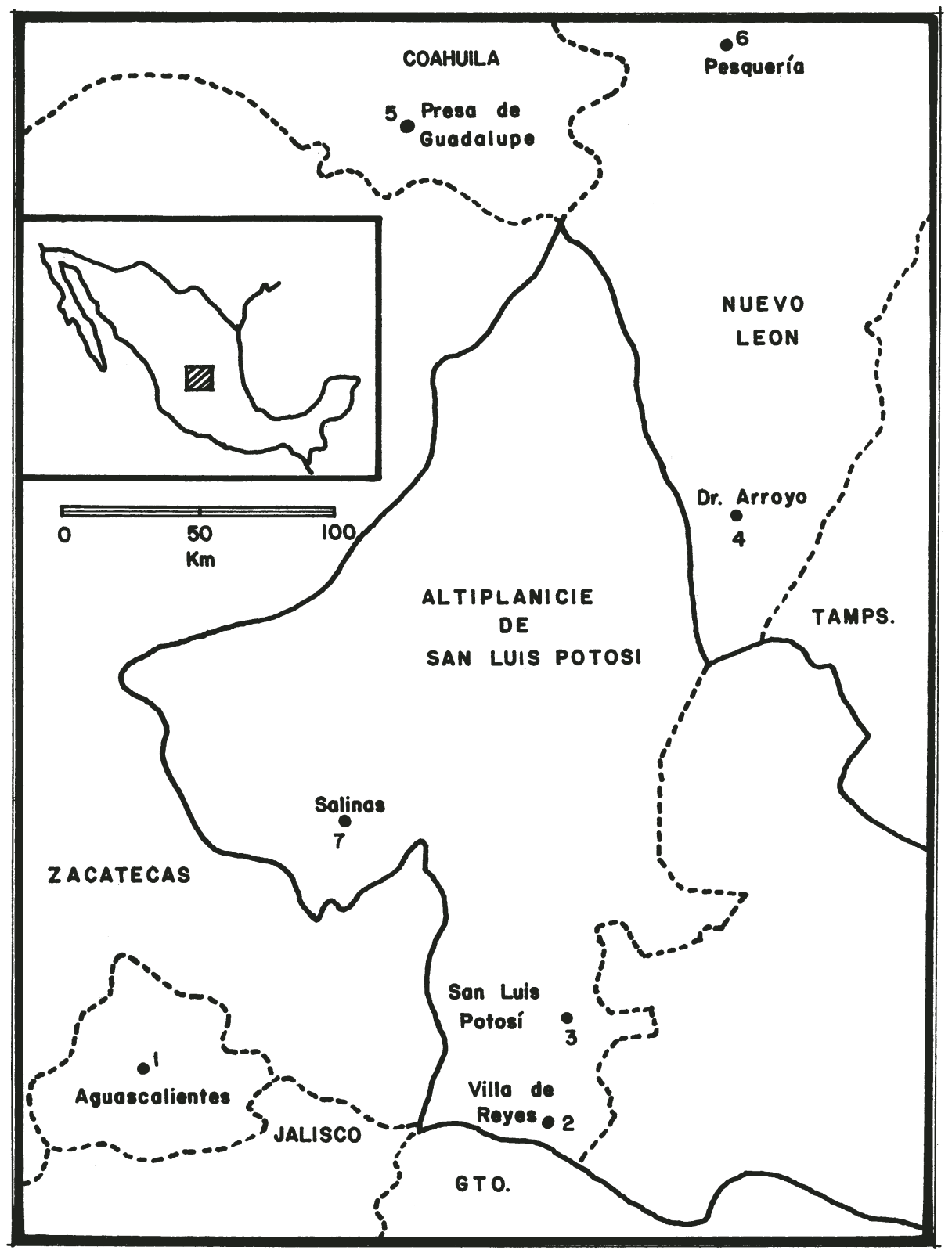

Fig. 1. Ubicación de los sitios de colecta. Los números $\hat{i}, 2,3$ y 4, corresponden a poblaciones de $P$. laevigata; en el sitio 5 prospera una población de $P$. glandulosa var. torreyana y en el sitio 7 crecen poblaciones mixtas de ambas especies. El sitio 6 corresponde a una población de $P$. glandulosa var. glandulosa. 


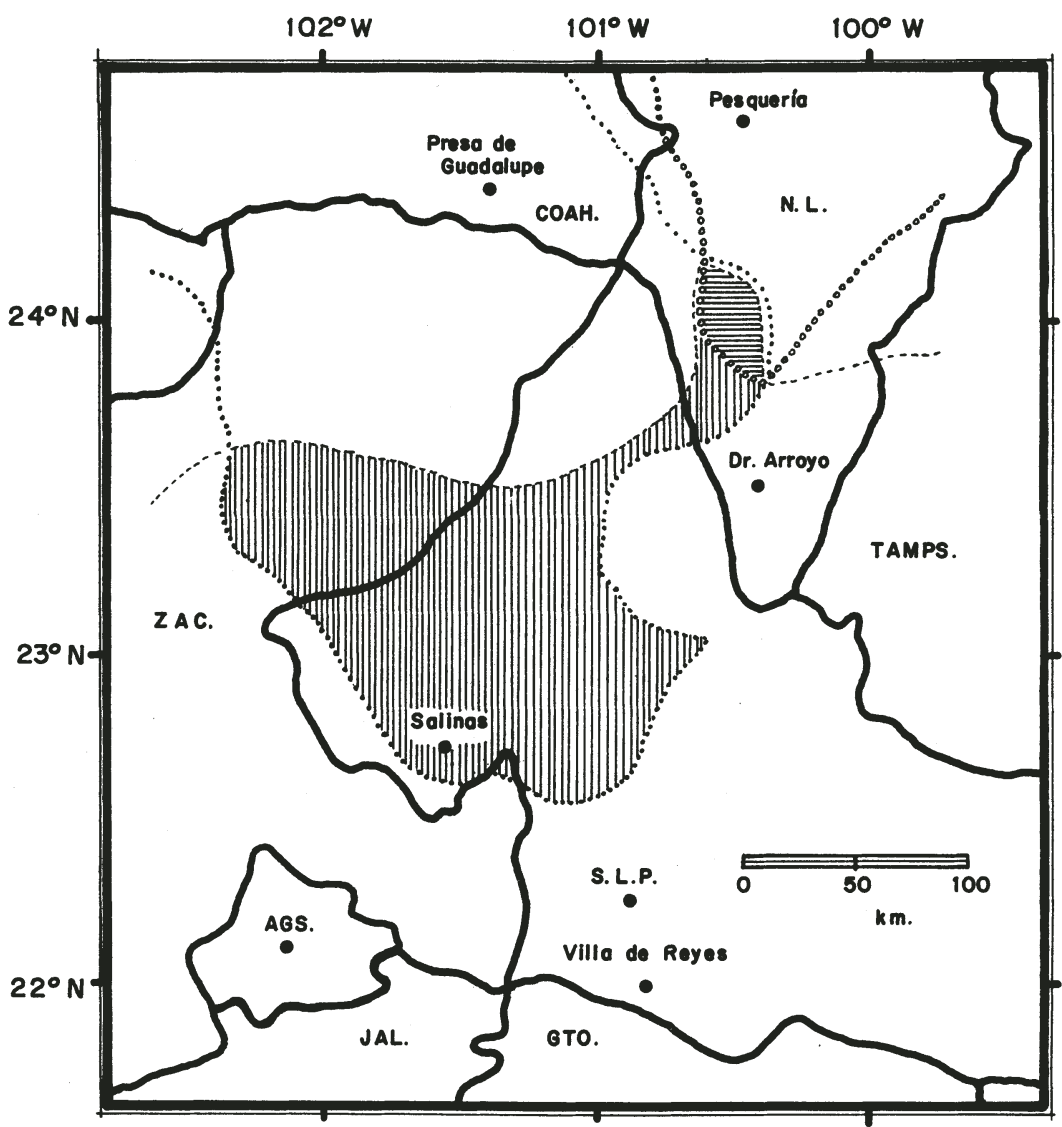

\section{SIMBOLOGIA}

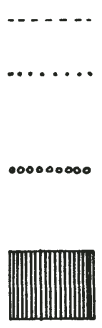

Limite norte de P. loevigato

Límite sur de P. glandulosa



Zona de simpatría entre $P$. var. torreyona.

glandulosa var. torreyana, $P$

Límite sur de P. glanduloso

laevigata y P. glandulosa

var. glandulosa.

var. glandulosa.

Limite de estados.

Zona de simpatria entre

P. laevigata y P. glandulosa

var. torreyana.

Fig. 2. Delimitación aproximada de las áreas de distribución de las especies de Prosopis en la altiplanicie de San Luis Potosí y regiones adyacentes. 
introgredidos. En este análisis se consideró una probabilidad umbral de 0.900 , de manera que si la probabilidad para alguna observación es menor al valor de la mencionada probabilidad umbral, dicha observación se clasificará en el grupo "otros".

Se sometio al análisis clasificatorio discriminadamente las observaciones del grupo 3 antes mencionado, proporcionándose como opciones los grupos 1, 2, 5 y "otros". Las 150 observaciones (75 colectadas) del grupo 3 (Salinas, S.L.P.), se clasificaron de la manera siguiente: en el grupo 1 fueron ubicadas 38 observaciones (25.3\%); en el grupo 2 se situaron 45 (30\%); en el grupo 5 se consideraron 30 observaciones (20\%), mientras que las restantes $37(26.7 \%)$ pasaron a constituir el grupo "otros".

$\mathrm{De}$ acuerdo con estos resultados, respaldados por la alta probabilidad umbral aplicada, las especies parentales (grupos 1 y 2) constituirlan en el sitio de colecta de Salinas $55.33 \%$ de los individuos de Prosopis. Los grupos 5 y "otros", en tanto, que conforman el faltante $44.67 \%$, incluirlan con mayor probabilidad a los individuos originados por hibridación e introgresión. Dadas sus características, el grupo 5 comprendería individuos híbridos $F 1$, mientras que el grupo "otros", además de híbridos $F 1$, contendría a los individuos originados por introgresión. La explicación se encuentra al considerar lo siguiente.

Respecto al modo de manifestación de la hibridación a nivel morfológico, los resultados consignados no difieren de lo señalado por Stace (1980). Este autor indica que habitualmente deberá esperarse que los híbridos posean una apariencia intermedia en relación a las especies parentales, pero agrega que ésto no siempre sucede, ya que la condición de intermedio se deja ver en muchos grados y maneras. Destaca también que la aparición de segregantes divergentes en la generación F2, representa una evidencia razonable de que los padres son híbridos, si bien la ausencia de segregantes no necesariamente revela que no ocurra hibridación. Respecto a ésto, Grant (1971) coincide. en señalar que mientras que la generación F1 suele ser intermedia en sus diversos atributos, la F2 y posteriores generaciones pueden mostrar una gran diversidad de tipos de recombinación. El grupo "otros" con toda probabilidad incluye individuos segregantes de la generación F2 y de generaciones subsiguientes.

Se ha observado en Salinas que de cualquier modo, los probables individuos de origen híbrido no se apartan mucho en su morfología de las especies parentales. De acuerdo con Grant (1971), ésto es de esperarse, ya que: "la híbridación introgresiva es una fuerza promotora de convergencia en características morfológicas y preferencias ecológicas en una zona de contacto simpátrico entre dos especies o semiespecies". De esta manera "...la mayoría de las oportunidades para el establecimiento de la progenie híbrida será para los genotipos que se aproximen a una especie parental o a la otra. La progenie producto de retrocruzas será entonces más apta para cumplir con los requisitos ecológicos, y, por lo tanto, le serán propicias las condiciones ambientales existentes".

\section{Discusión general}

Ninguna evidencia presentada en este estudio, por sí sola, constituye una prueba contundente de que exista hibridación entre $P$. laevigata y $P$. glandulosa var. torreyana. Reunidas, sin embargo, conforman una demostración de peso suficiente que permite afirmar con un alto grado de certidumbre que tal proceso se está dando. 
La severa perturbación antropogénica (explotación forestal, desmontes agrícolas, sobrepastoreo) a que se han visto sometidas las áreas de mezquital y, en general, los ecosistemas de la parte árida del país, desde la conquista española, ha representado sin duda un importante factor favorable para la hibridación en el mezquite. Stebbins (1957) y Grant (1971) señalan que las plantas híbridas sólo pueden llegar a establecerse si son adaptativamente superiores a los elementos parentales en el hábitat original, o bien si pueden establecerse en una hábitat diferente disponible. Agregan que la hibridación natural a menudo se ve restringida a ciertas localidades en las que el ambiente ha sido perturbado por el hombre.

En vista de que entre las especies de Prosopis aqui estudiadas casi no existen barreras en contra de la hibridación y la introgresión, cabe hacerse ahora la siguiente pregunta: ¿cómo es que hasta ahora han podido mantenerse en buen número los individuos puros de ambas especies? La probable capacidad de autofecundación de Prosopis descubierta en esta investigación permite explicarlo en parte. A la par, debe considerarse el hecho de que la hibridación sea probablemente un proceso historico relativamente reciente, favorecido, en buena medida, por las actividades antropogénicas posteriores a la conquista espanola.

\section{AGRADECIMIENTOS}

Se agradece al Comité Editorial de Acta Botánica Mexicana, asl como a la Dra. Beryl Simpson de la Universidad de Texas en Austin, sus valiosas observaciones y sugerencias.

\section{LITERATURA CITADA}

Anónimo. 1982. Statistical analysis systems (SAS) user's guide. SAS Institute Inc. Cary, North Carolina. $584 \mathrm{pp}$.

Arroyo, M. T. K. 1981. Breeding systems and pollination biology in Leguminosae. In: Polhill, R.M. \& P.H. Raven (eds.). Advances in legume systematics. Part 2. Royal Botanic Gardens. Kew. pp. 723769.

Benson, L. y R. A. Darrow. 1981. Trees and shrubs of the Southwestern Desert. 3a. ed. The University of Arizona Press. Tucson, Arizona. 416 pp.

Burkart, A. 1952. Las leguminosas argentinas, silvestres y cultivadas. 2a. ed. Acme. Buenos Aires. 569 pp.

Burkart, A. 1976. A monograph of the genus Prosopis (Leguminosae, subfam. Mimosoideae). Jour. Arnold Arbor. 57(3-4): 219-249, 450-525.

Felker, P. 1979. Mesquite, an all-purpose leguminous arid land tree. In: Ritchie, G.A. (ed.). New agricultural crops. American Association for the Advancement of Science. Selected Symposium 38. Westview Press. Boulder, Colorado. pp. 89-132.

Felker, P. 1981. Uses of tree legumes in semiarid regions. Econ. Bot. 35(2): 174-186.

Felker, P., G.H. Cannell, P.R. Clark, J.F. Osborn y P. Nash. 1983. Biomass production of Prosopis species (mesquite), Leucaena, and other leguminous trees grown under heat/drought stress. Forest Science 29(3): 592-602.

Galindo, S. 1983. Caracterización de la variación en el mezquite (Prosopis L.) y sus usos en el Altiplano Potosino. Tesis Profesional. Universidad Autónoma de Nuevo León. Monterrey, Nuevo León. 87 pp. 
Galindo, S. 1986. Hibridación natural en el mezquite (Prosopis laevigata y Prosopis glandulosa var. torreyana) de la Altiplanicie de San Luis Potosi. Tesis de Maestría en Ciencias. Centro de Botánica. Colegio de Postgraduados. Chapingo, México. 112 pp.

Galindo, S. y E. Garcia. 1986a. The uses of mesquite (Prosopis spp.) in the highlands of San Luis Potosí, México. Forest Ecology and Management 16:49-56.

Galindo, S. y E. García. 1986b. Usos del mezquite (Prosopis L.) en el Altiplano Potosino. Agrociencia 63:7-16.

Goodman, M. M. 1974. Numerical aids in taxonomy. In: Radford, A. et al. (eds.). Vascular plant systematics. Harper \& Row. New York. pp. 485-500.

Graham, J. D. 1960. Morphological variation in mesquite (Prosopis, Leguminosae) in the lowlands of northeastern México. The Southwestern Naturalist 5(4): 187-193.

Grant, V. 1971. Plant speciation. Columbia University Press. New York. 435 pp.

Hunziker, J. H., C. A. Naranjo, R. A. Palacios, L. Poggio y B. Saidman. 1986. Studies on the taxonomy, genetic variation and biochemistry of Argentine species of Prosopis. Forest Ecology and Management 16(1-4): 301-315.

Isely, D. 1972. Legumes of the United States. IV, Calliandra, Pithecellobium and Prosopis. Madroño 21(5): 287-298.

Jardine, N. y R. Sibson. 1971. Mathematical taxonomy. Wiley. London. 286 pp.

Johnston, M. C. 1962. The North American mesquites, Prosopis section Algarobia (Leguminosae). Brittonia 14: $72-90$.

Karlin, U. y R. Diaz. 1984. Potencialidad y manejo de algarrobos en el árido subtropical argentino. Ministerio de Educación y Justicia. Secretaría de Ciencia y Técnica. Programa Nacional de Recursos Naturales Renovables. Buenos Aires. $59 \mathrm{pp}$.

Leakey, R. y T. Last, 1980. Biology and potential of Prosopis species in arid environments, with particular reference to $P$. cineraria. Jour. Arid Environ. 3: 9-24.

Love, A. y D. Love. 1975. Plant chromosomes. Lubrecht \& Cramer. Leutherhausen. 184 pp.

Martínez, S. 1984. Arquitectura foliar de las especies del género Prosopis. Darwiniana 25(1-4): 279-297.

Mueller-Dombois, D. y H. Ellenberg. 1974. Aims and methods of vegetation ecology. Wiley. New York. 547 pp.

Nájera, F. y E. Pimienta. 1984. Factores que afectan el asentamiento de frutos en mezquite (Prosopis laevigata). In: Resúmenes del X Congreso Nacional de Fitogenética. Aguascalientes, Aguascalientes. p. 125.

Naranjo, C. A., L. Poggio, y S. E. Zeigger. 1984. Phenol chromatography, morphology and cytogenetics in three species and natural hybrids of Prosopis (Leguminosae-Mimosoideae). Plant Syst. Evol. 144: 257-276.

Orlóci, L. 1978. Multivariate analysis in vegetation research. 2a. ed. Dr. W. Junk Publishers. The Hague. $451 \mathrm{pp}$.

Palacios, R. A. y D. L. Bravo. 1975. Estudio morfológico de las semillas de Prosopis II: Algunas especies norteamericanas y neotropicales. Darwiniana 19(2-4): 357-372.

Palacios, R. A. y D. L. Bravo. 1981. Hibridación natural en Prosopis (Leguminosae) en la región chaqueña argentina. Evidencias morfológicas y cromatográficas. Darwiniana 23(1): 3-35.

Patiño, J. 1981. Manual para la elaboración de preparaciones cromosómicas en plantas. Universidad Autónoma Chapingo. Chapingo, México. 69 pp.

Signoret, P. J. 1970. Datos sobre algunas características ecológicas del mezquite (Prosopis laevigata) y su aprovechamiento en el Valle del Mezquital. In: Beltrán, E. (ed.). Mezquites y huizaches. Instituto Mexicano de Recursos Naturales Renovables. México, D.F. pp. 70-146.

Simpson, B., J. Neff y A. Modeke. 1977. Prosopis flowers as a resource. In: Simpson, B. (ed.) Mesquite: its biology in two desert ecosystems. Dowden, Hutchinson \& Ross. Stroudsberg, Pennsylvania. pp. 84-107.

Sokal, R. R. y P. H. Sneath. 1963. Principles of numerical taxonomy. W.H. Freeman. San Francisco, California. 359 pp. 
Solbrig, O. T., K. Bawa, N. Carman, J. H. Hunziker, C. A. Naranjo, R. A. Palacios, L. Poggio y B. B. Simpson. 1977. Patterns of variation. In: Simpson, B. (ed.). Mesquite: its biology in two desert ecosystems. Dowden, Hutchinson \& Ross. Stroudsburg, Pennsylvania. pp. 44-60.

Stace, C. A. 1980. Plant taxonomy and biosystematics. Arnold. London. 279 pp.

Stebbins, G. L. 1957. Variation and evolution in plants. 3a. ed. Columbia University Press. New York. $643 \mathrm{pp}$. 